\title{
Gravitational Faraday and spin-Hall effects of light
}

\author{
Andrey A. Shoom๑* \\ Max Planck Institute for Gravitational Physics (Albert Einstein Institute), Leibniz Universität Hannover, \\ Callinstr. 38, Hannover D-30167, Germany
}

(Received 23 March 2018; revised 21 October 2020; accepted 3 August 2021; published 1 October 2021)

\begin{abstract}
The gravitational Faraday and its dual spin-Hall effects of light arise in space-times of nonzero angular momentum. These effects were studied in stationary, asymptotically flat space-times. Here we study these effects in arbitrary, nonstationary, asymptotically flat space-times. These effects arise from the interaction between light polarization and space-time angular momentum. As a result of such interaction, the phase velocity of left- and right-handed circularly polarized light becomes different, that results in the gravitational Faraday effect. This difference implies different dynamics of these components, that begin to propagate along different paths-the gravitational spin-Hall effect of light. Due to this effect, the gravitational field splits a multicomponent beam of unpolarized light and produces polarized gravitational rainbow. The component separation is an accumulative effect observed in long range asymptotics. To study this effect, we construct uniform eikonal expansion and derive dynamical equation describing this effect. To analyze the dynamical equation, we present it in the local space and time decomposition form. The spatial part of the equation presented in the related optical metric is analogous to the dynamical equation of a charged particle moving in magnetic field under the influence of the Coriolis force.
\end{abstract}

DOI: 10.1103/PhysRevD.104.084007

\section{INTRODUCTION}

Gravitational field affects propagation of electromagnetic waves, in particular light, in different ways. For example, electromagnetic radiation emitted by hot accretion disk around a black hole into the external space gets gravitationally redshifted. Light rays passing by a strongly gravitating massive object, e.g., a star or a black hole, get deflected due to the space-time curvature in the vicinity of a massive object. The rays deflection depends on the object's mass and angular momentum. There is also the gravitational Faraday effect, analogical to the magneto-optical Faraday effect-a rotation of the polarization plane of a linearly polarized light propagating in a transparent material in the presence of a magnetic field along propagation of the light [1]. The gravitational Faraday effect is a rotation of the plane of polarization of an electromagnetic wave propagating in a stationary gravitational field, for example, near a stationary rotating black hole. The study and observation of this effect have quite long history (see, e.g., [2-15]). However, despite the clear analysis

\footnotetext{
*andrey.shoom@aei.mpg.de
}

Published by the American Physical Society under the terms of the Creative Commons Attribution 4.0 International license. Further distribution of this work must maintain attribution to the author(s) and the published article's title, journal citation, and DOI. Open access publication funded by the Max Planck Society. done in $[3,5]$, in some works rotation of the polarization vector around direction of light propagation, which is due to its coupling to the space-time angular momentum, is mixed with change of its direction due to deflection of the light rays.

It is known that in optics there is an effect dual to the Faraday effect- the optical Magnus effect- that results in the action of light polarization on its trajectory, causing its transverse polarization-dependent displacement. Due to this effect, a linearly polarized light splits into left- and right-handed circularly polarized components propagating along different paths. This splitting effect is known as the spin-Hall effect of light [16-20]. Spin-Hall effects became quite ubiquitous in modern physics. They are observed in condensed matter [21], optical [22], and high-energy systems [23]. Classical and quantum spin-Hall optical effects of light are described and analyzed in many works (see, e.g., [16-20,24-27].) The underlying nature of these effects is the spin-orbit interaction between the spin of a photon, an electron, or an atom, and its extrinsic angular momentum.

We may expect a dual to the gravitational Faraday effect-the gravitational spin-Hall effect of light. The gravitational Faraday and spin-Hall optical effects may not be surprising phenomena if one takes a certain point of view on the gravitational field. Namely, one can observe that the source-free Maxwell equations in curved spacetime, i.e., in the presence of gravity, can formally be considered as equations in flat space-time in the presence 
of a bianisotropic moving medium whose optical properties are defined by dielectric permittivity and magnetic permeability tensors expressed though the space-time metric tensor components. This point of view was proposed and developed already more than half-century ago by several authors $[2,3,28]$. There is also a dual point of view on propagation of light in the so-called metamaterials, whose optical properties can be synthesized on a subwavelength scale that allows us to control propagation of light in a nearly arbitrary way [29]. In this new field of transformation optics a metric approach can be used to calculate dielectric permittivity and magnetic permeability tensors of a metamaterial [30]. Another example is the gravitational analog of the linear magnetoelectric effect that was studied in [31].

Taking into account the analogy between a gravitational field and a bianisotropic moving medium, polarization of light was considered to describe its propagation in a stationary gravitational field. For example, by using the weak field approximation, it was shown that left- and right-handed circularly polarized light propagating near a rotating gravitational body get scattered in a different way [32-35]. To consider this effect in a strong stationary gravitational field, the so-called modified geometric optics formalism was introduced [36]. This formalism was applied to describe scattering of a polarized light propagating in the stationary space-time of a rotating (Kerr) black hole [37]. Later this approach was reformulated to some extent [38] and in a different context [39] in a four-dimensional covariant form.

There are different approaches have been taken to describe dynamics of polarized light in a curved space-time background (for a review see [40]). For instance, approach based on dynamics of massless spinning particle was proposed and developed in [41-44] for Riemannian and pseudo-Riemannian manifolds. A semiclassical approach to describe photon dynamics in a curved space-time background based on the Bargmann-Wigner equations was taken in [45], and helicity-dependent photon's evolution was predicted for the Schwarzschild space-time. Spin-Hall effect of light for the Schwarzschild spacetime was also predicted in [46]. However, in these works the proper orientation and propagation of the basis representing optic axes is not discussed. Thus it remains unclear how to measure properly the evolution of the light polarization along a null ray. As a result, the proposed effect is questionable. Such prediction is also contradictory to the analysis presented in, e.g., $[3,32,36,37,47]$, where it was shown that left- and righthanded polarization modes evolve differently due to the space-time angular momentum only, and in static spacetimes there is no distinction between propagation of these modes. In other words, absence of the gravitational Faraday effect in static space-times implies that these modes evolve in the same fashion.

To support a possibility of the spin-Hall effect of light in static space-times, one may appeal to the theoretical and experimental studies of the polarization-dependent deflection of light in a smoothly inhomogeneous isotropic medium, where such an effect was observed for light propagating through a planar (without torsion) optical fiber (see, e.g., [16,48]). Note, however, that according to [49] (and also the references therein) these polarizationdependent effects observed in planar curved optical fibers are of the higher order. It is also stated in [17] that there is no polarization-dependent ray shift can be observed in a planar waveguide, where the Rytov-Vladimirski-Berry phase [50-53], which determines rotation of the polarization plane, vanishes identically. We would also like to note that a gravitational field is analogical to a special kind of bianisotropic moving optical medium, such that there is no birefringence and its index of refraction is different for electromagnetic waves propagating in opposite directions [28]. ${ }^{1}$ In other words, a gravitational field is essentially different from a material optical anisotropic medium. Finally, we note that motion of the medium also makes significant contribution to observed optical phenomena and often gives rise to new effects, see, e.g., [55-58]. Detailed investigation of analogy between a material optical medium and a gravitational field in context of the related optical effects goes beyond the scope of this paper.

In this paper, to describe the gravitational Faraday and spin-Hall effects of light, we shall take the modified geometric optics approach [36] and extend it to arbitrary nonstationary asymptotically flat space-times of nonzero angular momentum [59]. In addition to stationary rotating black holes and stars, such space-times correspond to dynamical gravitational fields due to a gravitational collapse, black holes and neutron stars coalescence, and gravitational waves. They can also represent some cosmological models. Thus, these gravitational optical effects can be widely present. To describe properly these effects, the key property of a stationary space-time-existence of a timelike Killing vector field, was exploited. Here we extend these results by considering a field of static observers. The key property of such observers is that in their frame, at the spatial infinity $i^{0}$, the space-time total Arnowitt-DeserMisner (ADM) 3-momentum vanishes. The field of static observers naturally generalizes the field of Killing observers and coincides with it in stationary space-times.

This paper is organized as follows. In Sec. II we briefly review the laws of (canonical) geometric optics in a curved space-time. In the next section we study the gravitational Faraday effect of light in arbitrary (nonstationary) spacetime. In Sec. IV we define the field of static observers that can properly detect and measure the gravitational Faraday effect. In Sec. $\mathrm{V}$ we construct the uniform eikonal expansion that takes into account contribution of light

\footnotetext{
${ }^{1}$ One may try to describe this phenomenon in the language of Finsler geometry, using properties of the Randers metrics. For a nice review of the Finsler geometry and related problems see [54].
} 
polarization to its propagation and present dynamical equations that describe the gravitational spin-Hall effect of light, which is dual to the gravitational Faraday effect. The gravitational spin-Hall effect of light is presented in the local space and time decomposition form in Sec. VI. The last Sec. VII contains discussion of the derived results.

Here we shall use geometrized units $c=G=1$ and conventions adopted in the book [60].

\section{GEOMETRIC OPTICS}

Finding an exact electromagnetic wave solution to the Maxwell equations in a curved space-time background is a formidable problem. Moreover, often such solutions cannot be presented in a closed analytic form. However, for waves that are highly monochromatic over some space-time regions, an asymptotic short-wave (geometric optics) approximation can be used [60]. Such an approximation allows us to capture basic characteristics of light propagation in a curved space-time background defined by metric $g_{\alpha \beta}$ of the most general form.

The source-free Maxwell wave equation for the vector potential $A^{\alpha}$ in the Lorenz gauge

$$
A_{; \alpha}^{\alpha}=0
$$

reads

$$
-A_{; \beta}^{\alpha ; \beta}+R_{\beta}^{\alpha} A^{\beta}=0,
$$

where the semicolon stands for the covariant derivative associated with the space-time metric $g_{\alpha \beta}$ and $R_{\beta}^{\alpha}$ is the four-dimensional Ricci tensor. The geometric optics approach is based on splitting of the vector potential into a rapidly changing real phase, the eikonal $\theta$, and a slowly changing complex amplitude in the following way:

$$
A^{\alpha}=\Re\left\{\left(a^{\alpha}+\varepsilon b^{\alpha}+\cdots\right) e^{i \theta / \varepsilon}\right\},
$$

where $\varepsilon \ll 1$ is a dummy expansion parameter that helps to track order of terms: a term with $\varepsilon^{n}$, for some integer $n$, varies as $\left(\chi / l_{\min }\right)^{n}$, where $\chi / l_{\min } \ll 1$. Here $\chi$ is the reduced wavelength (wavelength/2 $\pi$ ) and $l_{\min }$ is the minimal of the two characteristic scales - the curvature radius of the wave front, or the length of a wave packet, and the local curvature radius of the space-time. Substituting the vector potential into the Lorentz gauge condition (1) and the wave equation (2) and collecting the leading terms of order $\varepsilon^{-2}$ and $\varepsilon^{-1}$ we derive the fundamental laws of geometric optics:

$$
\begin{gathered}
k^{\alpha} k_{\alpha}=0, \quad k^{\beta} k_{; \beta}^{\alpha}=0, \\
k^{\alpha} f_{\alpha}=0, \quad k^{\beta} f_{; \beta}^{\alpha}=0, \\
\left(a^{2} k^{\alpha}\right)_{; \alpha}=0 .
\end{gathered}
$$

Here $k^{\alpha}=d x^{\alpha} / d \lambda$ is the wave vector metrically related to the gradient $k_{\alpha} \equiv \theta_{; \alpha}$ and tangent to the light ray $\Gamma: x^{\alpha}=x^{\alpha}(\lambda)$, where $\lambda$ is affine parameter of the ray, $a \equiv\left(a^{\alpha} a_{\alpha}^{*}\right)^{1 / 2}$ is the scalar amplitude, and $f^{\alpha} \equiv a^{\alpha} / a$ is a unit complex polarization vector, such that $f^{\alpha} f_{\alpha}=0$ and $f^{\alpha} f_{\alpha}^{*}=1$. Here and in what follows the superscript $*$ stands for complex conjugation. These laws imply that light rays are the space-time null geodesics (4), the polarization vector is orthogonal to the light ray and parallel-propagated along it (5), and the vector $a^{2} k^{\alpha}$ is a conserved current, which defines the adiabatically conserved number of light rays, or in quantum language, the number of photons (6). The laws of geometric optics (4)-(6) reflect only an approximate picture of light propagation in a curved space-time. In this description polarization of light does not affect its path.

\section{GRAVITATIONAL FARADAY EFFECT OF LIGHT}

To measure angle of rotation of the polarization plane in the magneto-optical Faraday effect, we have to align properly optic axes of a polarizer and an analyzer. For example, we can align the polarizer and the analyzer at the polarizer's location and then parallel transport the analyzer along the ray trajectory. In a curved space-time this procedure is not so simple.

Let us present the polarization vector $f^{\alpha}$ in the following form: ${ }^{2}$

$$
f^{\alpha}=e^{i \varphi} m^{\alpha},
$$

where $m^{\alpha}$ is a unit complex vector, such that

$$
\begin{array}{r}
m^{\alpha} m_{\alpha}=0, \quad m^{\alpha} m_{\alpha}^{*}=1, \quad m^{\alpha} k_{\alpha}=0, \\
m^{\alpha} \equiv \frac{1}{\sqrt{2}}\left(e_{1}^{\alpha}+i \sigma e_{2}^{\alpha}\right), \quad m^{* \alpha} \equiv \frac{1}{\sqrt{2}}\left(e_{1}^{\alpha}-i \sigma e_{2}^{\alpha}\right),
\end{array}
$$

where $e_{1,2}^{\alpha}$ are real orthonormal space-like vectors. The local complex basis $\left\{m^{\alpha}, m^{* \alpha}\right\}$ plays a role of optic axes. To specify the polarization of a given wave we use the parameter $\sigma= \pm 1$, with "+" for the right- and "-" for the left-handed circularly polarized light [60]. This definition means that the polarization vector of the (left)right-handed circularly polarized light rotates in the (anti)clockwise direction, when viewed from the source.

To define a change in the rotation of the polarization vector along the light ray we introduce polarization phase $\varphi$. For example, in a vacuum and flat space-time $\varphi$ has a constant value in the basis $\left\{m^{\alpha}, m^{* \alpha}\right\}$ parallel transported along the ray. As we shall see, this polarization phase

\footnotetext{
${ }^{2}$ The polarization vector is defined modulo the wave vector $k^{\alpha}$. This gauge freedom does not affect the results that follow. We shall fix this gauge, as well as the rotation gauge transformation $m^{\alpha} \rightarrow m^{\alpha} \exp (i \psi)$, later.
} 
defines an additional angular shift of the polarization vector due to a gravitational field of nonzero angular momentum.

Using the propagation equation for the polarization vector (5) and the expression (7), we derive the propagation equation for the polarization phase along the null ray,

$$
k^{\alpha} \varphi_{; \alpha}=i m_{\alpha}^{*} k^{\beta} m_{; \beta}^{\alpha} .
$$

In a space-time decomposition, spatial part of this expression, corresponding to propagation of the polarization phase along the null ray trajectory, written in momentum parametrization, is the Rytov-Vladimirski-Berry phase [50-53]. This phase of light propagating in a helical optical fiber was experimentally measured and discussed in [61,62].

To compute the polarization phase for a given null ray, we have to define a propagation law for $m^{\alpha}$ along the ray. This can be done by an observer-defined local decomposition of the space-time into space and time. This is the so-called space-time threading approach, in contrast to the space-time slicing, which is known as the ADM approach. ${ }^{3}$

Consider a family of observers filling a three-dimensional space like a continuous medium. Each of the observers defines the local frame of reference. World lines of these observers form a congruence of integral curves of the timelike future directed unit vector field $u^{\alpha}=u^{\alpha}\left(x^{\alpha}\right)$, $u^{\alpha} u_{\alpha}=-1$. The local rest space $\Sigma_{u}$ orthogonal to $u^{\alpha}$ is a three-dimensional subspace of the tangent space defined at every event on an observer's world line. A vector from the tangent space can be projected into the subspace $\Sigma_{u}$ by means of the projection operator $p_{\beta}^{\alpha}=\delta_{\beta}^{\alpha}+u^{\alpha} u_{\beta}$, and $p_{\alpha \beta}=g_{\alpha \beta}+u_{\alpha} u_{\beta}$ defines the induced metric on $\Sigma_{u}$. Applying the projection operator to $k^{\alpha}$ we construct the unit spacelike vector $n^{\alpha}$ that defines the spatial direction of a light ray. Accordingly, we have

$$
k^{\alpha}=\omega\left(u^{\alpha}+n^{\alpha}\right),
$$

where $\omega \equiv-k_{\alpha} u^{\alpha}$ is the angular frequency of light measured by the local observer. This decomposition allows us to express propagation of $m^{\alpha}$ along $k^{\alpha}$ by defining its propagation along the vectors $u^{\alpha}$ and $n^{\alpha}$,

$$
k^{\beta} m_{; \beta}^{\alpha}=\omega\left(u^{\beta} m_{; \beta}^{\alpha}+n^{\beta} m_{; \beta}^{\alpha}\right) .
$$

We require that the basis vectors $m^{\alpha}$ and $m^{* \alpha}$ belong to $\Sigma_{u}$, and thus, according to (8) and (11), are orthogonal to $n^{\alpha}$. Because the polarization vector $f^{\alpha}$ is defined modulo $k^{\alpha}$, this requirement can be fulfilled at some event on the null ray. Then, as it is shown below, this orthogonality condition is preserved along the ray.

\footnotetext{
${ }^{3}$ The threading point of view was originally developed by Møller, Zelmanov, and Cattaneo, and discussed in detail in [63-65]. It is used in [66].
}

Next we construct a right-handed, observer-adapted orthonormal frame $\left\{e_{0}^{\alpha}, e_{a}^{\alpha}, a=1,2,3\right\}$, where $e_{0}^{\alpha} \equiv u^{\alpha}$, $e_{1,2}^{\alpha}$ are defined in (9), and $e_{3}^{\alpha} \equiv n^{\alpha}$. For such frame we have $\varepsilon_{\alpha \beta \gamma \delta} e_{0}^{\alpha} e_{1}^{\beta} e_{2}^{\gamma} e_{3}^{\delta}=+1$, where $\varepsilon_{\alpha \beta \gamma \delta}$ is the Levi-Civita (pseudo) tensor. Using (11), this gives

$$
\varepsilon_{\alpha \beta \gamma \delta} u^{\alpha} k^{\beta} m^{* \gamma} m^{\delta}=i \sigma \omega,
$$

which implies

$$
\varepsilon_{\alpha \beta \gamma \delta} m^{* \gamma} m^{\delta}=\frac{i \sigma}{\omega}\left(k_{\alpha} u_{\beta}-u_{\alpha} k_{\beta}\right) .
$$

We shall also need the following property of the Levi-Civita tensor:

$$
\varepsilon_{\mu \nu \gamma \delta} \varepsilon^{\mu \nu \alpha \beta}=-2\left(\delta_{\gamma}^{\alpha} \delta_{\delta}^{\beta}-\delta_{\delta}^{\alpha} \delta_{\gamma}^{\beta}\right),
$$

where $\delta_{\beta}^{\alpha}$ is the four-dimensional Kronecker tensor.

To measure properly the polarization phase, we require first that the basis $\left\{m^{\alpha}, m^{* \alpha}\right\}$ does not rotate with respect to a reference basis fixed at the spatial infinity, when it is spatially transported along a ray trajectory, ${ }^{4}$ and second that its initial orientation does not change when it is transported along the observer congruence. The first requirement is ensured by the vanishing spatial Fermi-Walker derivative of $m^{a}$ along $n^{a}$,

$$
\nabla_{n}^{\mathrm{FW}} m^{a} \equiv n^{b} m_{\mid b}^{a}-\left(a^{a} n^{b}-a^{b} n^{a}\right) m_{b}=0 .
$$

Here $a^{a}=n^{b} n^{a}{ }_{b b}$ and the stroke $\mid$ stands for the covariant derivative associated with the spatial metric $p_{a b}=e_{a}^{\alpha} e_{b}^{\beta} p_{\alpha \beta}$, such that $p_{a b \mid c}=0$. This derivative is related to the covariant derivative associated with the space-time metric $g_{\alpha \beta}$ as follows: $e_{a}^{\alpha} n^{b} m^{a}{ }_{\mid b}=p_{\gamma}^{\alpha} n^{\beta} m_{; \beta}^{\gamma}$. The orthogonality condition $m^{\alpha} n_{\alpha}=m^{a} n_{a}=0$ is preserved by the Fermi-Walker derivative. To fulfill the second requirement, we impose that the basis $\left\{m^{\alpha}, m^{* \alpha}\right\}$ is corotating with the congruence. This implies that the basis has no relative temporal rotation with respect to nearby observers and, as a result, with respect to the reference basis fixed at the spatial infinity. This requirement is ensured by the vanishing temporal corotating FermiWalker derivative of $m^{\alpha}$ along $u^{\alpha}$ (see, e.g., [63]),

$\nabla_{u}^{\mathrm{CFW}} m^{\alpha} \equiv u^{\beta} m_{; \beta}^{\alpha}+\left(w^{\alpha} u^{\beta}-w^{\beta} u^{\alpha}\right) m_{\beta}-\omega_{\beta}^{\alpha} m^{\beta}=0$.

Here $w^{\alpha}=u^{\beta} u^{\alpha}{ }_{\beta}$ is 4-acceleration and $\omega_{\alpha \beta}=p_{\alpha}^{\gamma} p_{\beta}^{\delta} u_{[\gamma ; \delta]}$ is the vorticity tensor. The first three terms represent the temporal Fermi-Walker derivative. The orthogonality condition $m^{\alpha} u_{\alpha}=0$ is preserved by the corotating FermiWalker derivative. The conditions (16) and (17) fix the

\footnotetext{
${ }^{4}$ For description of optical measurements in curved space-time see, e.g., [67].
} 
scalar function $\psi=\psi\left(x^{\alpha}\right)$ in the gauge transformation $m^{\alpha} \rightarrow m^{\alpha} \exp (i \psi)$.

Using the decomposition of the null vector $k^{\alpha}(11)$, the expressions (12), (14), (15), and the transport laws (16) and (17), we can calculate the right-hand side of (10) as follows,

$$
i m_{\alpha}^{*} k^{\beta} m_{; \beta}^{\alpha}=i \omega m^{* \alpha} \omega_{\alpha \beta} m^{\beta}=\sigma \omega^{\alpha} k_{\alpha}
$$

where

$$
\omega^{\alpha}=\frac{1}{2} \varepsilon^{\alpha \beta \gamma \delta} u_{\beta} \omega_{\gamma \delta}=\frac{1}{2} \varepsilon^{\alpha \beta \gamma \delta} u_{\beta} u_{\gamma ; \delta}
$$

is the vorticity of the observers congruence. ${ }^{5}$

By using this result, we can now compute the polarization phase $\varphi$ for a given null ray $\Gamma: x^{\alpha}=x^{\alpha}(\lambda)$,

$$
\varphi=\sigma \int_{\Gamma} \omega^{\alpha} k_{\alpha} d \lambda=\sigma \int_{\Gamma} \omega_{\alpha} d x^{\alpha} .
$$

Finally, we can consider a linearly polarized light, viewed as a superposition of its left- and right-handed circularly polarized components. The linear polarization real unit vector $f_{L}^{\alpha}=\left(f^{\alpha}+f^{* \alpha}\right) / \sqrt{2}$ rotates with respect to the basis $\left\{m^{\alpha}, m^{* \alpha}\right\}$ and the angle of rotation $\varphi_{L}$ measured along the light ray $\Gamma$ is

$$
\varphi_{L}=\int_{\Gamma} \omega_{\alpha} d x^{\alpha} .
$$

This rotation is known as the gravitational Faraday effect of light.

\section{FIELD OF OBSERVERS}

So far we have not specified the field of observers $u^{\alpha}$. As it follows from the expressions (20) and (21), the gravitational Faraday rotation depends on vorticity of the observers congruence. For example, freely falling (inertial) observers do not feel the gravitational field and their congruence has zero vorticity. The same situation happens for the zero angular momentum observers. Thus, such observers do not detect the gravitational Faraday rotation. Alternatively, we can consider a congruence of arbitrarily moving (noninertial) observers whose congruence has nonzero vorticity. Such observers would claim to detect the gravitational Faraday rotation in a flat space-time. What kind of observers one has to consider in order to measure properly the gravitational Faraday effect?

The polarization-dependent gravitational optical effects were studied in stationary space-times. Such space-times posses timelike Killing vector field $\xi_{(t)}^{\alpha}$, where $t$ is the

\footnotetext{
${ }^{5}$ For a hypersurface-orthogonal vector field $u^{\alpha}$ vorticity is identically zero. In the next section we define a field of static observers whose vorticity is not identically zero.
}

Killing time, a parameter along Killing vector field orbits. Naturally, in such space-times the field of Killing observers was taken, $u^{\alpha} \propto \xi_{(t)}^{\alpha}$. Here we consider asymptotically flat nonstationary space-times (see, e.g., [68]). Such spacetimes do not possess timelike Killing vector field. In this case we use the following construction. Take an inertial frame in the asymptotically flat region and construct connected to the frame Cartesian coordinate latticework. Such a latticework is assumed to be absolutely rigid and extends to other regions of the space-time as far as possible. ${ }^{6}$ Place identical clocks in every point of the latticework and synchronize them modulo the redshift factor, i.e., (proper time of a point on the latticework) $=$ (redshift factor at that point) $\times$ (proper time on the latticework at the asymptotically flat region). This construction represents a field of observers that are situated at every point of the latticework, i.e., they have fixed spatial coordinate position defined by asymptotically Cartesian coordinates: ( $x^{i}=$ const, $\left.i=1,2,3\right)$. Accordingly, in these coordinates the observers field is

$$
u^{\alpha}=\frac{1}{\sqrt{h}} \delta_{0}^{\alpha} .
$$

Here $h>0$ is the squared redshift factor and $x^{0}=t$ is the timelike coordinate that measures proper time of observers sitting on the latticework in the asymptotically flat region. Using a timelike threading approach [66] we can present the space-time metric in the coordinates $\left(x^{0}=t, x^{i}\right)$ in the following form:

$$
d s^{2}=-h\left(d t-g_{i} d x^{i}\right)^{2}+h \gamma_{i j} d x^{i} d x^{j}
$$

Here $h \gamma_{i j}$ is the three-dimensional metric that defines spatial distance and the metric functions $h, g_{i}$, and $\gamma_{i j}$ depend on $t$ and $x^{i}$. Accordingly, the covariant form of the observers field (22) reads

$$
u_{\alpha}=-\sqrt{h}\left(\delta_{\alpha}^{0}-g_{i} \delta_{\alpha}^{i}\right)
$$

Let us now compute the vorticity (19) of the observers field (24) in the metric (23),

$\omega^{\alpha}=\frac{1}{2 h}\left\{(\boldsymbol{g}, \operatorname{curl} \boldsymbol{g}) \delta_{0}^{\alpha}+\left(\left[\boldsymbol{g} \times \boldsymbol{g}_{, t}^{\mathrm{b}}\right]^{i}+(\operatorname{curl} \boldsymbol{g})^{i}\right) \delta_{i}^{\alpha}\right\}$.

Here $\boldsymbol{g}^{\mathrm{b}}$ is the covariant form $g_{i}$ of the vector $\boldsymbol{g}$ living in a three-dimensional space endowed with the metric $\gamma_{i j}$, and

\footnotetext{
${ }^{6}$ Note, however, that such a latticework cannot be extended into certain space-time regions, for example into a rotating black hole's ergosphere or into a black hole interior.
} 


$$
\begin{aligned}
(\boldsymbol{a}, \boldsymbol{b}) & =a^{i} b^{j} \gamma_{i j}, \quad[\boldsymbol{a} \times \boldsymbol{b}]_{i}=e_{i j k} a^{j} b^{k}, \\
(\operatorname{curl} \boldsymbol{g})^{i} & =e^{i j k} g_{k, j}, \quad e_{i j k}=\sqrt{\gamma} \epsilon_{i j k}, \quad e^{i j k}=\frac{\epsilon^{i j k}}{\sqrt{\gamma}},
\end{aligned}
$$

where $\gamma=\operatorname{det}\left(\gamma_{i j}\right), \quad \epsilon_{123}=\epsilon^{123}=1$ is the Levi-Civita symbol, and the indices are raised and lowered by $\gamma_{i j}$. The expressions $(\ldots)_{, t}$ and $(\ldots)_{, i}$ mean partial derivatives of (...) with respect to $t$ and $x^{i}$. Using (25) we can calculate the gravitational Faraday rotation (21).

The problem is that the observers field $u^{\alpha}$ is not unique. One can consider another field of observers $\tilde{u}^{\alpha^{\prime}}$ that have fixed spatial coordinate position on the related latticework $\left(x^{i^{\prime}}, i^{\prime}=1^{\prime}, 2^{\prime}, 3^{\prime}\right)$. This new lattice work and the proper time $x^{0^{\prime}}=t^{\prime}$ of such observers located at the asymptotically flat infinity are related to the former ones by the Lorentz transformation, $x^{\alpha}=\Lambda_{\beta^{\prime}}^{\alpha} x^{\beta^{\prime}}$ (see, e.g., [60], p. 69), and the above expressions (22)-(24) have the same form in the primed frame. To understand how the gravitational Faraday rotation depends on the observers field, one has to find how the vorticity expressions corresponding to $u^{\alpha}$ and $\tilde{u}^{\alpha^{\prime}}$ are related to each other. To do this, let us first derive a relation between the observers vector fields $u^{\alpha}$ and $\tilde{u}^{\alpha^{\prime}}$ in the frame $x^{\alpha}$, which is

$$
\tilde{u}^{\alpha}=\frac{\gamma_{g}}{1-(\boldsymbol{g}, \mathbf{v})}\left\{u^{\alpha}+\frac{v^{i}}{\sqrt{h}} \delta_{i}^{\alpha}\right\},
$$

where

$$
\gamma_{g} \equiv \frac{1}{\sqrt{1-\left(\mathbf{v}_{g}, \mathbf{v}_{g}\right)}}, \quad \mathbf{v}_{g} \equiv \frac{\mathbf{v}}{1-(\mathbf{v}, \boldsymbol{g})},
$$

and $\mathbf{v}$ is the 3 -velocity with the constant contravariant components ( $v^{i}=$ const, $i=1,2,3$ ), which are parameters of the Lorentz transformation. ${ }^{7}$ Accordingly, the covariant form of the new observers field (27) reads

$$
\tilde{u}_{\alpha}=-\gamma_{g} \sqrt{h}\left(\delta_{\alpha}^{0}-\tilde{g}_{i} \delta_{\alpha}^{i}\right),
$$

where

$$
\tilde{\boldsymbol{g}} \equiv \boldsymbol{g}+\mathbf{v}_{g}
$$

Now we can compute the vorticity (19) of the observers field (29) in the metric (23),

$\tilde{\omega}^{\alpha}=\frac{\gamma_{g}^{2}}{2 h}\left\{(\tilde{\boldsymbol{g}}, \operatorname{curl} \tilde{\boldsymbol{g}}) \delta_{0}^{\alpha}+\left(\left[\tilde{\boldsymbol{g}} \times \tilde{\boldsymbol{g}}_{, t}^{b}\right]^{i}+(\operatorname{curl} \tilde{\boldsymbol{g}})^{i}\right) \delta_{i}^{\alpha}\right\}$.

A comparison between the expressions (25) and (31) shows that $\tilde{\omega}^{\alpha} \neq \omega^{\alpha}$. This implies that the gravitational Faraday effect (20), (21) is observer-dependent. In particular, in a

\footnotetext{
${ }^{7}$ In a curved space-time region $v_{i}=\gamma_{i j} v^{j} \neq$ const.
}

static space-time, such that in the frame $x^{\alpha}$ we have $g_{i}=0$, one can find an observers field of nonzero vorticity. Such observers would claim to detect the gravitational Faraday effect proportional to $v^{i}$, i.e., to the parameters of the Lorentz transformation. In the next section we study the gravitational spin-Hall effect of light, which is dual to the gravitational Faraday effect. This dual effect is, in turn, also proportional to $v^{i}$. However, in a static space-time, for $v^{i}=0$ both the effects vanish. Note that this phenomenon is analogical to the relativistic Hall effect resulting in a transverse shift of the relativistic center of inertia of a dynamical system [69]. The shift is proportional to the intrinsic angular momentum of the system and to the velocity $v^{i}$ of the relativistic frame, which is moving with respect to the rest frame of the system. The key issue behind the relativistic Hall effect is that components of the threedimensional vector of the relativistic center of inertia are not spatial components of a four-dimensional vector. Thus, they do not transform in covariant way, which makes the location of the relativistic center of inertia frame dependent [66]. In our case, the analogical vector is the three-dimensional vector $\boldsymbol{g}$, which transforms according to (30).

Thus, in the case of a nonstatic gravitational field $\left(g_{i} \neq 0\right)$, the general field of observers (27) can detect the gravitational Faraday rotation due to both the gravitational field and their own motion, i.e., via the values of $v^{i}$. This observer-dependent problem is resolved in stationary space-times by selecting the preferred field of Killing observers. Our goal is to find a field of observers that is analogical to the Killing observers in an arbitrary, nonstationary space-time. In other words, we have to fix the kinematic gauge freedom $v^{i}$, that brings us back to the question raised in the first paragraph of this section.

To answer the question, we note first that an asymptotically flat stationary space-time has vanishing total 3-momentum, as defined with respect to the observers field that coincides with the field of Killing observers. We can take this property as the property that allows us to fix the kinematic gauge and thus to define the observers field in nonstationary space-times. To begin, we recall that asymptotic flatness structure allows the space-time energymomentum 4-vector $P^{\alpha}=\left(E, P^{i}\right)$ to be well defined at the spatial infinity $i^{0}$ as follows (for more details see [68], Ch. 11 and the references therein). We consider globally hyperbolic space-times. A globally hyperbolic space-time can be foliated by Cauchy hypersurfaces $\Sigma_{t}$ parametrized by a global time function $t$. Consider a unit, timelike, future-directed vector field $N_{\alpha} \propto t_{; \alpha}$. Then, the space-time metric $g_{\alpha \beta}$ induces a three-dimensional spatial metric

$$
H_{\alpha \beta}=g_{\alpha \beta}+N_{\alpha} N_{\beta}
$$

on each $\Sigma_{t}$. Let $\Sigma_{t}$ be such that this metric at $i^{0}$ in the asymptotically Cartesian coordinates $\left(x^{i}, i=1,2,3\right)$ has 
the form $\delta_{i j}+\mathcal{O}(1 / r)$, where $\delta_{i j}$ is the three-dimensional Kronecker tensor and $r=\sqrt{x^{i} x_{i}}$. Then, the space-time total energy $E$ and 3-momentum $P_{i}$ are defined as follows:

$$
\begin{gathered}
E \equiv \frac{1}{16 \pi} \lim _{r \rightarrow \infty} \oint_{\mathcal{S}}\left(H_{i j, i}-H_{i i, j}\right) S^{j} d A, \\
P_{i} \equiv \frac{1}{8 \pi} \lim _{r \rightarrow \infty} \oint_{\mathcal{S}}\left(K_{i j} S^{j}-K_{j}^{j} S_{i}\right) d A,
\end{gathered}
$$

where summation over repeated indices is assumed. The integrals are taken over a 2 -sphere $\mathcal{S}: r=$ const, $S^{i}$ is a unit, outward-directed, spacelike vector orthogonal to $\mathcal{S}, d A$ is the area element on $\mathcal{S}$, which in the limit $r \rightarrow \infty$ and in spherical coordinates $(r, \theta, \phi)$ takes the form $d A=$ $r^{2} \sin ^{2} \theta d \theta d \phi$, and

$$
\begin{aligned}
K_{i j} & \equiv \frac{1}{2}\left(\mathcal{L}_{N} \boldsymbol{H}\right)_{i j} \\
& =\frac{1}{2}\left(N^{k} H_{i j, k}+H_{k j} N_{, i}^{k}+H_{i k} N_{, j}^{k}\right),
\end{aligned}
$$

is the hypersurface extrinsic curvature. In this construction, the so-called ADM energy-momentum 4-vector

$$
P_{\alpha}=-E N_{\alpha}+P_{i} \delta_{\alpha}^{i}
$$

is independent of the choice of $\Sigma_{t}$. As a result, the spacetime total energy $E$ and total 3-momentum $\boldsymbol{P}$ depend only on the asymptotic behavior of a spacelike hypersurface $\Sigma_{t}$ at $i^{0}$ and transform under Lorentz boost as the components of a 4 -vector. Thus, by an appropriate choice of the $t$ function, that is, by taking a proper boost at the asymptotic spatial infinity $i^{0}$, one can make the space-time total 3-momentum $\boldsymbol{P}$ vanish. This choice of $t$ fixes the kinematic gauge and defines the corresponding field of observers, which we shall call static observers. The field of static observers naturally generalizes the field of Killing observers, which is hypersurface orthogonal at asymptotic infinity. Static observers coincide with Killing observers in stationary space-times.

In what follows, to discuss the gravitational Faraday effect and its dual gravitational spin-Hall effect, we shall always consider the field of static observers. According to the conditions (16) and (17), these observers possess an unidirectional basis $\left\{m^{\alpha}, m^{* \alpha}\right\}$, adjusted to a reference basis fixed at the spatial infinity, that allows them to measure properly these optical effects.

\section{GRAVITATIONAL SPIN-HALL EFFECT OF LIGHT}

As we already noted, in the geometric optics approach polarization does not affect light rays. A similar situation occurs when one applies the Wentzel-Kramers-Brillouin (WKB) method to the Dirac equation: electric and magnetic particle's moments and spin do not affect its trajectory [70,71]. However, the WKB expansion is not uniformly valid in its domain. At finite fixed distances from inhomogeneous field regions, effects of the particle's moments on its trajectory are of order $\hbar$ and they vanish in the classical limit $\hbar \rightarrow 0$. In this case, the WKB method gives correct result. But for distances of order $\hbar^{-1}$, the effects become of order unity and do not vanish in the limit $\hbar \rightarrow 0$. In this case, the WKB method fails. As it was explained in [71], to obtain an expansion which is uniformly valid everywhere, including the neighborhood of infinity, one has to include effects of the particle's moments and spin on its trajectory $[49,72,73]$. Analogously, to have an eikonal expansion uniformly valid everywhere, one has to take into account contribution of internal degrees of freedom (polarization) to propagation of light [36]. Such a contribution is of order $\varepsilon$ for short distances of propagation. However, it accumulates along light ray trajectory and for sufficiently large distances (of order $\varepsilon^{-1}$ ) it becomes of order $\varepsilon^{0}$.

To construct such an expansion we have to include polarization phase into the eikonal. As we already found, in the geometric optics approximation,

$$
A^{\alpha} \approx \mathfrak{R}\left\{a^{\alpha} e^{i \theta / \varepsilon}\right\}, \quad a^{\alpha}=a m^{\alpha} e^{i \varphi} .
$$

Here the polarization phase $\varphi$ changes along light ray trajectory in accordance with (20), but this change does not affect the trajectory. Our goal is to modify the light trajectory in accordance with the polarization phase change. Let us rewrite this expression in the following form:

$$
A^{\alpha} \approx \mathfrak{R}\left\{a m^{\alpha} e^{i(\theta+\varepsilon \varphi) / \varepsilon}\right\}
$$

This form suggests us to define the modified eikonal:

$$
\tilde{\theta} \equiv \theta+\varepsilon \tilde{\varphi},
$$

where $\tilde{\varphi}$ is the modified polarization phase that corresponds to the modified eikonal $\tilde{\theta}$.

With these modifications the expression (38) reads:

$$
A^{\alpha} \approx \mathfrak{R}\left\{\tilde{a}^{\alpha} e^{i \tilde{\theta} / \epsilon}\right\}
$$

Here the amplitude $\tilde{a}^{\alpha}$ corresponds to the modified eikonal (39). Substituting this approximation into the Lorenz gauge condition (1) and the wave equation (2) we derive the following leading order equations:

$$
\mathrm{k}^{\alpha} \mathrm{k}_{\alpha}=0, \quad \tilde{a}_{\alpha} \mathrm{k}^{\alpha}=0 .
$$

They imply that the wave vector

$$
\mathrm{k}_{\alpha} \equiv \theta_{; \alpha}=\tilde{\theta}_{; \alpha}-\varepsilon \tilde{\varphi}_{; \alpha}
$$


is null and electromagnetic waves are transverse. These conditions hold along null ray defined by the wave vector. The term $\tilde{\varphi}_{; \alpha}$ is the gradient of the polarization component of the modified eikonal. For a particular null ray $\tilde{\Gamma}: \tilde{x}^{\alpha}=\tilde{x}^{\alpha}(\lambda)$, with the wave vector $\mathrm{k}^{\alpha}=d \tilde{x}^{\alpha} / d \lambda$, the polarization phase is [cf. (20)],

$$
\tilde{\varphi}=\sigma \int_{\tilde{\Gamma}} \omega_{\alpha} d \tilde{x}^{\alpha}=\sigma \int_{\tilde{\Gamma}} \omega^{\alpha} \mathrm{k}_{\alpha} d \lambda .
$$

The gradient of the polarization component is

$$
\tilde{\varphi}_{; \alpha}=\sigma \omega_{\alpha}
$$

To construct the propagation equation for the wave vector, we use the same method which is used in the geometric optics (see [60], p. 576). Namely, taking the covariant derivative of the first expression in (41) we derive $\mathrm{k}^{\beta} \mathrm{k}_{\beta ; \alpha}=0$, while the expression (42) and the equality $\tilde{\theta}_{; \beta \alpha}=\tilde{\theta}_{; \alpha \beta}$ give

$$
\mathrm{k}_{\beta ; \alpha}=\mathrm{k}_{\alpha ; \beta}-\varepsilon \sigma \Phi_{\alpha \beta},
$$

where we defined

$$
\Phi_{\alpha \beta} \equiv \omega_{\beta ; \alpha}-\omega_{\alpha ; \beta} .
$$

The expression (45) gives us the null rays equation

$$
\mathrm{k}^{\beta} \mathrm{k}_{; \beta}^{\alpha}=\varepsilon \sigma \Phi_{\beta}^{\alpha} \mathrm{k}^{\beta} .
$$

Note that this equation looks like the Lorentz force law. ${ }^{8}$

Now we shall construct propagation equation for the amplitude $\tilde{a}^{\alpha}$. We begin with the transversality condition [the second expression in (41)]. This condition should hold along a null ray, $\mathrm{k}^{\beta}\left(\tilde{a}_{\alpha} \mathrm{k}^{\alpha}\right)_{; \beta}=0$. Expanding this expression and using (47) we derive

$$
\mathrm{k}^{\alpha} \mathrm{k}^{\beta} \tilde{a}_{\alpha ; \beta}=\varepsilon \sigma \Phi_{\alpha \beta} \mathrm{k}^{\alpha} \tilde{a}^{\beta} .
$$

The right-hand side of this expression is of order $\varepsilon$. The corresponding order complement comes from the subleading order $\varepsilon^{-1}$ wave equation (2),

$$
\mathrm{k}^{\beta} \tilde{a}_{\alpha ; \beta}=-\frac{1}{2} \tilde{a}_{\alpha} \mathrm{k}_{; \beta}^{\beta}-i \tilde{a}_{\alpha} \mathrm{k}^{\beta} \tilde{\varphi}_{; \beta} .
$$

\footnotetext{
${ }^{8}$ Equation (47) can formally be derived by taking the ultrarelativistic limit $m \rightarrow 0$ of the Lorentz force law, where $m$ is the rest mass of a charged particle and its proper time is defined as $\tau \equiv m \lambda$ [37]. In this analogy the product of the particle's charge and the electromagnetic field tensor $F_{\alpha \beta}$ corresponds to $\varepsilon \sigma \Phi_{\alpha \beta}$ and $\omega_{\alpha}$ plays the role of an electromagnetic vector potential. Note that in analogy with the action for a charged particle moving in an electromagnetic field, Eq. (47) can also be derived from the generalized Fermat's principle [74-77].
}

Combining these expressions we derive the propagation equation for the complex amplitude $\tilde{a}^{\alpha}$,

$$
\mathrm{k}^{\beta} \tilde{a}_{; \beta}^{\alpha}=\varepsilon \sigma \Phi_{\beta}^{\alpha} \tilde{a}^{\beta}-\frac{1}{2} \tilde{a}^{\alpha} \mathrm{k}_{; \beta}^{\beta}-i \tilde{a}^{\alpha} \mathrm{k}^{\beta} \tilde{\varphi}_{; \beta} .
$$

Now we multiply this equation by the complex conjugate amplitude $\tilde{a}^{* \alpha}$ and add to it its complex conjugate form multiplied by $\tilde{a}^{\alpha}$. As a result, we derive

$$
\left(\tilde{a}^{2} \mathrm{k}^{\alpha}\right)_{; \alpha}=0
$$

where $\tilde{a} \equiv\left(\tilde{a}^{\alpha} \tilde{a}_{\alpha}^{*}\right)^{1 / 2}$ is the scalar amplitude. The derived equation implies adiabatic conservation of photons propagating along null curves defined by (47). To complete the construction of equations corresponding to the modified eikonal (39) we introduce the unit complex polarization vector $\mathrm{f}^{\alpha} \equiv \tilde{a}^{\alpha} / \tilde{a}$, such that $\mathrm{f}^{\alpha} \mathrm{f}_{\alpha}=0$ and $\mathrm{f}^{\alpha} \mathrm{f}_{\alpha}^{*}=1$. Then, the second expression in (41) and the expression (50) give

$$
\mathrm{k}^{\alpha} \mathrm{f}_{\alpha}=0
$$

$$
\mathrm{k}^{\beta} \mathrm{f}_{; \beta}^{\alpha}=\varepsilon \sigma \Phi_{\beta}^{\alpha} \mathrm{f}^{\beta}-i \mathrm{f}^{\alpha} \mathrm{k}^{\beta} \tilde{\varphi}_{; \beta} .
$$

The expressions (41), (47), (51), (52), and (53) represent the modified geometric optics corresponding to the modified eikonal (39). The last step is to show that in the limit $\varepsilon \rightarrow 0$ polarization phase of $f^{\alpha}$ does not change along the modified null rays. To begin with we present the polarization vector $\mathrm{f}^{\alpha}$ in the form [cf. (7)]

$$
\mathrm{f}^{\alpha}=e^{i \psi \mathrm{m}^{\alpha}} .
$$

Here $\psi$ is measured with respect to the complex basis $\left\{\mathrm{m}^{\alpha}, \mathrm{m}^{* \alpha}\right\}$, which is analogical to the previously considered basis $\left\{m^{\alpha}, \bar{m}^{\alpha}\right\}$ (see Sec. III). Accordingly, one can repeat the steps in Sec. III and derive [cf. (18)]

$$
i \mathrm{~m}_{\alpha}^{*} \mathrm{k}^{\beta} \mathrm{m}_{; \beta}^{\alpha}=\sigma \omega^{\alpha} \mathrm{k}_{\alpha}
$$

Then, from the relations (44), (53), (54), and (55), it follows that

$$
\mathrm{k}^{\alpha} \psi_{; \alpha}=\frac{\varepsilon}{2 \omega} \varepsilon_{\alpha \beta \gamma \delta} u^{\alpha} \mathrm{k}^{\beta} \Phi^{\gamma \delta} .
$$

Thus, as we already noted, the change of the polarization phase along the modified light ray is of order $\varepsilon$. This implies that the internal degree of freedom, the order $\varepsilon^{0}$ polarization phase $\varphi$, was indeed included into the modified eikonal.

Using the modified eikonal (42), we can compute phase velocity of each polarization component defined with respect to its geometric optics null ray, 


$$
v_{\mathrm{ph}} \equiv-\frac{\mathrm{k}_{\alpha} u^{\alpha}}{\mathrm{k}_{\alpha} n^{\alpha}}=\frac{\omega}{\omega-\varepsilon \sigma \omega_{\alpha} n^{\alpha}} .
$$

This expression implies that the phase velocity of left- and right-handed circularly polarized light is different. This difference results in the gravitational Faraday effect discussed in Sec. III.

Let us now consider the dynamical equation for light rays (47). Its right-hand side depends on light polarization $\sigma= \pm 1$ and represents the force of interaction between the light polarization and space-time angular momentum. This force is orthogonal to the wave vector $\mathrm{k}^{\alpha}$. This type of polarization-dependent force is a manifestation of the gravitational spin-Hall effect of light: the back-reaction of the changing polarization phase onto light trajectory results in its transverse, polarization-dependent displacement. As a result of such displacement, a linearly polarized beam of light propagating in a gravitational field of nonzero angular momentum along the space-time null geodesic will split into components of left- and right-hand circular polarizations and each component will propagate along a different null world line. These world lines get gradually displaced away in the opposite directions from the null geodesic. The group velocity of these components, which is the locally measured Poynting vector divided by the electromagnetic energy density, is equal to speed of light in vacuum. According to the generalized Fermat's principle [74-77], the expression (67) in the next section, integrated along a light ray trajectory, is stationary for null geodesics with respect to null variations. Thus, the propagation time $t$ for these components is greater than that of the corresponding null geodesic.

In the next section we shall discuss the gravitational spinHall effect in local space and time decomposition. To conclude, we recall that source-free Maxwell equations in a four-dimensional space-time are conformally invariant. Accordingly, the expressions above are invariant with respect to the conformal transformation of the metric $g_{\alpha \beta}=\Omega^{2} \bar{g}_{\alpha \beta}$ accompanied by the following conformal transformations:

$$
\begin{gathered}
\mathrm{k}^{\alpha}=\Omega^{-2} \overline{\mathrm{k}}^{\alpha}, \quad u^{\alpha}=\Omega^{-1} \bar{u}^{\alpha}, \\
\tilde{a}^{2}=\Omega^{-2} \overline{\tilde{a}}^{2}, \quad \mathrm{f}^{\alpha}=\Omega^{-1} \overline{\mathrm{f}}^{\alpha}+\kappa \Omega^{-2} \overline{\mathrm{k}}^{\alpha},
\end{gathered}
$$

where the scalar function $\kappa$ solves equation

$$
\overline{\mathrm{k}}^{\alpha} \kappa_{, \alpha}+i \kappa \overline{\mathrm{k}}^{\alpha} \tilde{\varphi}_{; \alpha}+\overline{\mathrm{f}}^{\alpha} \Omega_{; \alpha}=0,
$$

while $\omega_{\alpha}$ and $\Phi_{\alpha \beta}$ are conformally invariant, and the local angular frequency of light transforms as $\omega=\Omega^{-1} \bar{\omega}$.

\section{LOCAL SPACE AND TIME DECOMPOSITION}

Let us now present the dynamical equation (47) in a local decomposition defined by a static observer's 4-velocity $u^{\alpha}$. To simplify our computations, we will work in the metric $\bar{g}_{\alpha \beta}$ conformally related to the space-time metric (23) via the conformal factor $\Omega^{2}=h$. In what follows, we shall drop the bar signs. Because of the conformal invariance of the dynamical equation, our final expressions will be valid in the original space-time metric $g_{\alpha \beta}$, assuming that all dynamical quantities are transformed accordingly, as shown in the end of the previous section.

To compute the expression (46) we need the covariant form of the vorticity vector [see (25) and the text below],

$$
\omega_{\alpha}=\delta_{\alpha}^{i} A_{i}, \quad \boldsymbol{A}=\frac{1}{2}\left(\left[\boldsymbol{g} \times \boldsymbol{g}_{, t}^{\mathrm{b}}\right]+\operatorname{curl} \boldsymbol{g}\right) .
$$

Now we can calculate $\Phi_{\alpha \beta}$,

$$
\Phi_{0 i}=-\mathcal{E}_{i}, \quad \Phi_{i j}=e_{i j k} \mathcal{B}^{k},
$$

where we defined

$$
\mathcal{E} \equiv-\boldsymbol{A}_{, t}, \quad \mathcal{B} \equiv \operatorname{curl} \boldsymbol{A} .
$$

The three-dimensional fields $\mathcal{E}$ and $\mathcal{B}$ can be considered as "electric" and "magnetic" components of $\Phi_{\alpha \beta}$.

The next step is to apply the projection operators $-u^{\alpha} u_{\beta}$ and $p_{\beta}^{\alpha}=\delta_{\beta}^{\alpha}+u^{\alpha} u_{\beta}$ to the dynamical equation. These operators project four-dimensional objects onto $u^{\alpha}$ and its local orthogonal three-dimensional hypersurface $\Sigma_{u}$, which gives their local space and time decomposition. However, it is more convenient to use the decomposition of the wave vector $\mathrm{k}^{\alpha}=\omega\left(u^{\alpha}+\mathrm{n}^{\alpha}\right)$ [cf. (11)], where the light ray frequency $\omega=-\mathrm{k}^{\alpha} u_{\alpha}$ is measured by a local static observer and the unit spacelike vector $\mathrm{n}^{\alpha}$, orthogonal to $u^{\alpha}$, defines spatial direction of the light ray. Then, we contract the expression (45) with $u^{\beta}$ and $\mathrm{n}^{\beta}$ separately and apply the projection operators. Contraction with $u^{\beta}$ gives us an expression lying entirely in $\Sigma_{u}$ and involving time derivatives of the frequency and the unit spacelike vector. Contraction with $\mathrm{n}^{\beta}$ followed by projection onto $u^{\alpha}$ gives us (naturally) the same expression contracted with $\mathrm{n}^{\alpha}$,

$$
\left(1+g_{i} \mathrm{n}^{i}\right) \omega_{, t}+\mathrm{n}^{i} \omega_{, i}+\frac{\omega}{2}\left(2 \mathrm{n}^{i} g_{i, t}+\mathrm{n}^{i} \mathrm{n}^{j} \gamma_{i j, t}\right)=\varepsilon \sigma \mathcal{E}_{i} \mathrm{n}^{i}
$$

Finally, projecting the expression (45) contracted with $\mathrm{n}^{\beta}$ onto $\Sigma_{u}$ and using a local triad $e_{a}^{\alpha}$ defined on $\Sigma_{u}$ gives us the light ray dynamical equation on $\Sigma_{u}$,

$$
\begin{gathered}
\mathrm{n}^{b} \mathrm{n}_{\mid b}^{a}=\frac{\omega_{, b}}{\omega} \Pi^{a b}+2[\mathrm{n} \times \boldsymbol{A}]^{a}+\frac{\varepsilon \sigma}{\omega}\left[\mathrm{n} \times \mathcal{B}_{g}\right]^{a}, \\
\mathcal{B}_{g} \equiv \mathcal{B}+[\mathcal{E} \times \boldsymbol{g}] .
\end{gathered}
$$


Here $\mathrm{n}^{b} \mathrm{n}^{a}{ }_{b}$ is the covariant derivative defined in the metric $\gamma_{a b}=e_{a}^{i} e_{b}^{j} \gamma_{i j}$ along a light ray trajectory with the unit tangent vector $\mathrm{n}^{\alpha}=d x^{\alpha} / d l=e_{a}^{\alpha} \mathrm{n}^{a}$, where $d l=\omega d \lambda$ is the proper distance in the three-dimensional hyperspace $\Sigma_{u}$, and $\Pi^{a b}=\gamma^{a b}-\mathrm{n}^{a} \mathrm{n}^{b}$ is the three-dimensional projection operator onto a local two-dimensional subspace orthogonal to $\mathrm{n}^{a}$. The proper distance $d l$ is related to the time coordinate $t$ as follows:

$$
\frac{d t}{d l}=1+g_{i} \mathrm{n}^{i},
$$

where the scalar product is defined in the metric $\gamma_{a b}$. The expressions (64), (65), and (67) represent the local space and time decomposition of the dynamical equation (47).

Let us now analyse the derived result. Our main object is the dynamical ray equation (65). To understand better its physical meaning, we introduce the optical metric $\pi_{a b}$ and the three-dimensional wave vector $\mathbb{k}_{a}$ as follows

$$
\pi_{a b} \equiv \omega^{2} \gamma_{a b}, \quad \mathbb{k}_{a} \equiv \omega \mathrm{n}_{a} .
$$

Then, the dynamical ray equation (65) takes the following form:

$$
\frac{D \mathbb{k}}{d \ell}=2[\mathbb{k} \times \boldsymbol{A}]+\frac{\varepsilon \sigma}{\omega}\left[\mathbb{k} \times \mathcal{B}_{g}\right] .
$$

Here $D \mathbb{k} / d \ell$ is the covariant derivative of the wave vector defined in the optical metric, $d \ell=\omega d l$ is the optical length, and the vector products are defined in the optical metric. This equation is similar to the dynamical equation of a charged particle moving in a noninertial rotating frame in the presence of a magnetic field, i.e., the term $2[\mathbb{k} \times \boldsymbol{A}]$ is the Coriolis force and the next term is the Lorentz force. This last term is the transverse, polarization-dependent force that gives rise to the gravitational spin-Hall effect of light. This force depends on light frequency. Thus, the resultant splitting of a nonmonochromatic beam of light onto left- and right-handed circularly polarized components is frequency dependent. Namely, the low frequency part of the beam gets more deflected in the transversal direction, as compared to the high frequency part. As a result of such deflection, we have a polarized gravitational rainbow.

To conclude, we remark that in the case of a stationary space-time, our static observers are Killing observers and the results above reduce to those derived in [36].

\section{DISCUSSION}

Here we presented the study of the gravitational Faraday and spin-Hall effects of light in arbitrary, nonstationary, asymptotically flat space-time of nonzero angular momentum. This is a generalization of the modified geometric optics formalism developed in [36] and applied to propagation of polarized light in the stationary space-time of a rotating (Kerr) black hole [37]. The key concept of this generalization is the field of static observers defined in Sec. IV. In a stationary space-time, this field of observers naturally reduces to the field of Killing observers. The field of observers is uniquely defined in accordance with vanishing space-time ADM 3-momentum. The gravitational Faraday and spin-Hall effects are described properly by such static observers. One may try to explore the formalism developed here in the language of Finsler geometry and the related Randers metric applied to stationary spacetimes in [78].

The natural limitation of our formalism is breakdown of the geometric optics applicability and the concept of the static observers field. As we already pointed out in Sec. IV (see footnote 6), such observers cannot exist in space-time regions where a rigid static latticework spreading out of asymptotically flat region cannot be defined, for example in ergoregions or black hole interior. Let us also note that detection of the gravitational Faraday and spin-Hall effects of light requires measurements of a very high sensitivity. These effects are due to strong nonstatic gravitational fields and they accumulate during light propagation. The angular split of left- and right-handed circularly polarized light components is proportional to the space-time angular momentum [32-35,37], whereas spatial separation of these components is an accumulative effect and proportional to the propagation distance. Thus, the effect may not easily be observable in weak gravitational fields and relatively small spatial regions, e.g., within the Solar System [34], but it could potentially be detected in the light emerged from strongly gravitating systems, such as the vicinity of a black hole, and propagated sufficiently large distance.

\section{ACKNOWLEDGMENTS}

The author would like to thank the anonymous referee for bringing paper [65] to his attention.
[1] L. D. Landau and E. M. Lifshitz, Electrodynamics of Continuous Media: Course of Theoretical Physics, Volume 8 (Pergamon Press, Ltd., Headington Hill Hall, Oxford, England, 1975).
[2] G. V. Skrotskii, On the influence of gravity on the light propagation, Akad. Nauk SSR, Dokl. 114, 73 (1957) [Sov. Phys. Dokl. 2, 226 (1957)]. 
[3] J. Plebanski, Electromagnetic waves in gravitational fields, Phys. Rev. 118, 1396 (1960).

[4] B. B. Godfrey, Mach's principle, the Kerr metric, and blackhole physics, Phys. Rev. D 1, 2721 (1970).

[5] S. Pineault and R. C. Roeder, Applications of geometrical optics to the Kerr metric. I. Analytical results, Astrophys. J. 212, 541 (1977).

[6] P. A. Connors and R. F. Stark, Observable gravitational effects on polarised radiation coming from near a black hole, Nature (London) 269, 128 (1977).

[7] P. A. Connors, T. Piran, and R. F. Stark, Polarization features of X-ray radiation emitted near black holes, Astrophys. J. 235, 224 (1980).

[8] F. Fayos and J. Llosa, Gravitational effects on the polarization plane, Gen. Relativ. Gravit. 14, 865 (1982).

[9] H. Ishihara, M. Takahashi, and A. Tomimatsu, Gravitational Faraday rotation induced by a Kerr black hole, Phys. Rev. D 38, 472 (1988).

[10] P. Carini, L. L. Feng, and M. Li, R. Ruffini, Phase evolution of the photon in Kerr spacetime, Phys. Rev. D 46, 5407 (1992).

[11] M. Nouri-Zonoz, Gravitoelectromagnetic approach to the gravitational Faraday rotation in stationary space-times, Phys. Rev. D 60, 024013 (1999).

[12] M. Sereno, Gravitational Faraday rotation in a weak gravitational field, Phys. Rev. D 69, 087501 (2004).

[13] M. Sereno, Detecting gravitomagnetism with rotation of polarization by a gravitational lens, Mon. Not. R. Astron. Soc. 356, 381 (2005).

[14] A. Brodutch, T. F. Demarie, and D. R. Terno, Photon polarization and geometric phase in general relativity, Phys. Rev. D 84, 104043 (2011).

[15] T. Ghosh and A. K. Sen, The effect of gravitation on the polarization state of a light ray, Astrophys. J. 833, 82 (2016).

[16] V. S. Liberman and B. Y. Zel'dovich, Spin-orbit interaction of a photon in an inhomogeneous medium, Phys. Rev. A 46, 5199 (1992).

[17] K. Y. Bliokh and Y. P. Bliokh, Modified geometrical optics of a smoothly inhomogeneous isotropic medium: The anisotropy, Berry phase, and the optical Magnus effect, Phys. Rev. E 70, 026605 (2004).

[18] K. Y. Bliokh and Y. P. Bliokh, Topological spin transport of photons: The optical magnus effect and Berry phase, Phys. Lett. A 333, 181 (2004).

[19] K. Y. Bliokh and Y. P. Bliokh, Conservation of Angular Momentum, Transverse Shift, and Spin Hall Effect in Reflection and Refraction of Electromagnetic Wave Packet, Phys. Rev. Lett. 96, 073903 (2006).

[20] K. Y. Bliokh, Geometrodynamics of polarized light: Berry phase and spin Hall effect in a gradient-index medium, J. Opt. A: Pure Appl. Opt. 11, 094009 (2009).

[21] D. Xiao, M. C. Chang, and Q. Niu, Berry phase effects on electronic properties, Rev. Mod. Phys. 82, 1959 (2010).

[22] M. Onoda, S. Murakami, and N. Nagaosa, Hall Effect of Light, Phys. Rev. Lett. 93, 083901 (2004).

[23] A. Berard and H. Mohrbach, Spin Hall effect and Berry phase of spinning particles, Phys. Lett. A 352, 190 (2006).

[24] K. Y. Bliokh, A. Niv, V. Kleiner, and E. Hasman, Geometrodynamics of spinning light, Nat. Photonics 2, 748 (2008).
[25] K. Bliokh, Y. Gorodetski, V. Kleiner, and E. Hasman, Spin-Orbit Interaction in Optics: Coriolis Effect and Unified Geometric Phase, Phys. Rev. Lett. 101, 030404 (2008).

[26] K. Y. Bliokh, F. J. Rodríguez-Fortuño, F. Nori, and A. V. Zayats, Spin-orbit interactions of light, Nat. Photonics 9, 796 (2015).

[27] K. Y. Bliokh, D. Smirnova, and F. Nori, Quantum spin Hall effect of light, Science 348, 1448 (2015).

[28] A. M. Volkov, A. A. Izmestev, and G. V. Skrotskii, The propagation of electromagnetic waves in a Riemannian space, Zh. Eksp. Teor. Fiz. 59, 1254 (1970) [Sov. Phys. JETP 32, 686 (1971)].

[29] V. M. Shalaev, Transforming light, Science 322, 384 (2008).

[30] R. A. Crudo and J. G. O'Brien, Metric approach to transformation optics, Phys. Rev. A 80, 033824 (2009).

[31] G. W. Gibbons and M. C. Werner, The gravitational magnetoelectric effect, Universe 5, 88 (2019).

[32] B. Mashhoon, Scattering of electromagnetic radiation from a black hole, Phys. Rev. D 7, 2807 (1973).

[33] B. Mashhoon, Electromagnetic scattering from a black hole and the glory effect, Phys. Rev. D 10, 1059 (1974).

[34] B. Mashhoon, Can Einstein's theory of gravitation be tested beyond the geometrical optics limit?, Nature (London) 250, 316 (1974).

[35] B. Mashhoon, Influence of gravitation on the propagation of electromagnetic radiation, Phys. Rev. D 11, 2679 (1975).

[36] V. P. Frolov and A. A. Shoom, Spinoptics in a stationary space-time, Phys. Rev. D 84, 044026 (2011).

[37] V. P. Frolov and A. A. Shoom, Scattering of circularly polarized light by a rotating black hole, Phys. Rev. D 86, 024010 (2012).

[38] C. M. Yoo, Notes on spinoptics in a stationary spacetime, Phys. Rev. D 86, 084005 (2012).

[39] S. R. Dolan, Higher-order geometrical optics for electromagnetic waves on a curved spacetime, arXiv:1801.02273.

[40] M. A. Oancea, C. F. Paganini, J. Joudioux, and L. Andersson, An overview of the gravitational spin Hall effect, arXiv: 1904.09963.

[41] C. Duval, Z. Horváth, and P. A. Horváthy, Fermat principle for spinning light, Phys. Rev. D 74, 021701(R) (2006).

[42] C. Duval, Z. Horváth, and P. A. Horváthy, Geometrical spinoptics and the optical Hall effect, J. Geom. Phys. 57, 925 (2007).

[43] C. Duval, Polarized spinoptics and symplectic physics, arXiv:1312.4486.

[44] C. Duval and T. Schucker, Gravitational birefringence of light in Robertson-Walker cosmologies, Phys. Rev. D 96, 043517 (2017).

[45] P. Gosselin, A. Bérard, and H. Mohrbach, Spin Hall effect of photons in a static gravitational field, Phys. Rev. D 75, 084035 (2007).

[46] M. A. Oancea, J. Joudioux, I. Dodin, D. Ruiz, C. F. Paganini, and L. Andersson, The gravitational spin Hall effect of light, Phys. Rev. D 102, 024075 (2020).

[47] L. C. S. Leite, S. R. Dolan, and L. C. B. Crispino, Absorption of electromagnetic and gravitational waves by Kerr black holes, Phys. Lett. B 774, 130 (2017). 
[48] A. V. Dooghin, N. D. Kundikova, V. S. Liberman, and B. Ya. Zel'dovich, Optical Magnus effect, Phys. Rev. A 45, 8204 (1992).

[49] Yu. A. Kravtsov, O. N. Naida, and A. A. Fuki, Waves in weakly anisotropic 3D inhomogeneous media: Quasiisotropic approximation of geometrical optics, Sov. Phys. Usp. 39, 129 (1996).

[50] S. M. Rytov, Dokl. Akad. Nauk SSSR 18, 263 (1938); reprinted in Topological Phases in Quantum Theory, edited by B. Markovski and S. I. Vinitski (World Scientific, Singapore, 1989).

[51] V. V. Vladimirski, Dokl. Akad. Nauk SSSR 31, 222 (1941); reprinted in Topological Phases in Quantum Theory, edited by B. Markovski and S. I. Vinitski (World Scientific, Singapore, 1989).

[52] M. V. Berry, Quantal phase factors accompanying adiabatic changes, Proc. R. Soc. Lond. A 392, 45 (1984).

[53] S. I. Vinitskii, V. L. Derbov, V. M. Dubovik, B. L. Markovski, and Yu. P. Stepanovskii, Topological phases in quantum mechanics and polarization optics, Sov. Phys. Usp. 33, 403 (1990).

[54] G. W. Gibbons and C. M. Warnick, The Geometry of sound rays in a wind, Contemp. Phys. 52, 197 (2011).

[55] A. M. Khromykh, Ring generator in a rotating reference system, Zh. Eksp. Teor. Fiz. 50, 281 (1966) [Sov. Phys. JETP 23, 185 (1966)].

[56] A. M. Volkov and V. A. Kiselev, Proper frequencies of a rotating ring resonator, Zh. Eksp. Teor. Fiz. 57, 1353 (1969) [Sov. Phys. JETP 30, 733 (1970)].

[57] V. E. Privalov and Yu. V. Filatov, Investigation of the output characteristic of a rotating ring gas laser, Kvanotvaya Elektron. (Moscow) 4, 1418 (1977); Sov. J. Quantum Electron. 7, 802 (1977).

[58] B. M. Bolotovskii and S. N. Stolyarov, Current status of the electrodynamics of moving media (infinite media), Sov. Phys. Usp. 17, 875 (1975).

[59] A. Ashtekar, Asymptotic structure of the gravitational field at spatial infinity, in General Relativity and Gravitation, edited by A. Held (Plenum, New York, 1980), Vol. 2, p. 37.

[60] C. W. Misner, K. S. Thorne, and J. A. Wheeler, Gravitation (W. H. Freeman and Co., San Francisco, 1973).

[61] R. Y. Chiao and Y.S. Wu, Manifestations of Berry's Topological Phase for the Photon, Phys. Rev. Lett. 57, 933 (1986).
[62] A. Tomita and R. Y. Chiao, Observation of Berry's Topological Phase by Use of an Optical Fiber, Phys. Rev. Lett. 57, 937 (1986); Erratum, Phys. Rev. Lett. 57, 2471 (1986).

[63] R. T. Jantzen, P. Carini, and D. Bini, The many faces of gravitoelectromagnetism, Ann. Phys. (N.Y.) 215, 1 (1992).

[64] P. Carini and R. T. Jantzen, Gravitoelectro-magnetism and the single gyro, in Relativistic Gravitational Experiments in Space, edited by M. Demianski and C. W. F. Everitt (World Scientific Publishing Co. Pte. Ltd., Singapore, 1993), p. 135 .

[65] S. Boersma and T. Dray, Slicing, threading and parametric manifolds, Gen. Relativ. Gravit. 27, 319 (1995).

[66] L. D. Landau and E. M. Lifshitz, The Classical Theory of Fields: Course of Theoretical Physics Volume 2 (Pergamon Press, Ltd., Headington Hill Hall, Oxford, England, 1975).

[67] J. L. Synge, Optical observations in general relativity, Seminario Mat. Fis. di Milano 30, 271 (1960).

[68] R. M. Wald, in General Relativity (The University of Chicago Press, Ltd., London, 1984), p. 293.

[69] K. Y. Bliokh and F. Nori, Relativistic Hall Effect, Phys. Rev. Lett. 108, 120403 (2012).

[70] W. Pauli, Diracs Wellengleichung des Elektrons und geometrische Optik, Helv. Phys. Acta 5, 179 (1932).

[71] S. I. Rubinow and J. B. Keller, Asymptotic solution of the Dirac equation, Phys. Rev. 131, 2789 (1963).

[72] R. Schiller, Quasi-classical theory of the spinning electron, Phys. Rev. 125, 1116 (1962).

[73] R. Schiller, Quasi-classical theory of a relativistic spinning electron, Phys. Rev. 128, 1402 (1962).

[74] V. Perlick, On Fermat's principle in general relativity. I. The general case, Classical Quantum Gravity 7, 1319 (1990).

[75] V. Perlick, On Fermat's principle in general relativity. II. The conformally stationary case, Classical Quantum Gravity 7, 1849 (1990).

[76] R. Nityananda and J. Samuel, Fermat's principle in general relativity, Phys. Rev. D 45, 3862 (1992).

[77] V. P. Frolov, Generalized Fermat's principle and action for light rays in a curved spacetime, Phys. Rev. D 88, 064039 (2013).

[78] G. W. Gibbons, C. A. R. Herdeiro, C. M. Warnick, and M.C. Werner, Stationary metrics and optical ZermeloRanders-Finsler geometry, Phys. Rev. D 79, 044022 (2009). 